\title{
L'école à la maison au Québec : l'expression d'un choix familial marginal
}

Christine Brabant

Faculté d'éducation, Université de Sherbrooke

Sylvain Bourdon

Faculté d'éducation, Université de Sherbrooke

France Jutras

Faculté d'éducation, Université de Sherbrooke

\section{Résumé}

L'éducation à domicile est une option éducative marginale, quoique légale et présente à travers le monde. Au Québec, jusqu'ici, aucune étude approfondie n'avait décrit cette pratique et la population des familles concernées était inconnue. Cet article porte sur les raisons du choix de l'«école à la maison», exprimées par 203 familles québécoises au moyen d'un questionnaire. Cette enquête, réalisée en 2003, visait à documenter les conceptions de l'éducation, les raisons du choix, l'expérience éducative et les caractéristiques sociodémographiques des familles. Les raisons évoquées sont multiples et hétérogènes. Les rationnels décisionnels des parents sont variés et multidimensionnels. Une des particularités de ces résultats est qu'aucune voix religieuse, anti-étatique ou philosophique ne semble dominer le discours des parents-éducateurs québécois. Pour l'ensemble des participants, les principaux facteurs à la base de ce choix sont un désir de poursuivre un projet éducatif familial, une objection aux modes d'organisation $d u$ système scolaire, une volonté d'offrir de l'enrichissement et un souci du développement socioaffectif des enfants.

\begin{abstract}
Home schooling is a borderline, though legal, option that is exercised throughout the world. Until now, there has been no in-depth study of this practice in Quebec and there has been no head count of the families concerned. This article deals with the reasons for choosing home schooling, as given by 203 Quebec families who responded to a questionnaire on the subject. The survey, carried out in 2003, was designed to document the families 'representation of education, the reasons for their choice, their educational experience and their socio-demographic profiles. The reasons they gave were both numerous and diversified. The rationale of the parents' decisions is both varied and multidimensional. One of the particularities of the results is that the discourse of these Quebec parental educators seems quite devoid of any religious, anti-State or philosophical overtones. For the participants as a whole, the basic factors that largely
\end{abstract}


determined their choice were their desire to implement a family educational project, their objection to the modes of organization of the school system, a desire to offer an enriched learning experience and a concern with the socio-affective development of their children.

\section{Introduction}

L'éducation des enfants sous la responsabilité des parents, à domicile et dans la communauté, est la forme d'éducation qui fut pratiquée pendant la plus grande partie de l'histoire de l'humanité. Les services de précepteurs, de tuteurs et d'écoles spécialisées ont, à certains moments, complété l'éducation que les parents souhaitaient pour leurs enfants. Dans un effort d'éducation des masses et de maintien de l'ordre civil, les institutions religieuses puis publiques ont rendu l'instruction accessible aux enfants de toutes les classes sociales. Depuis à peine un peu plus de cent ans dans le monde et depuis 1943 au Québec, les sociétés modernes se sont dotées de lois établissant l'obligation de fréquentation scolaire pour les enfants et les adolescents. L'offre éducative des écoles publiques et privées ne rencontrant pas les attentes de toutes les familles, plusieurs pays ont prévu dans leur législation une possibilité d'exemption de cette obligation, permettant ainsi aux parents de conserver l'entière responsabilité de l'éducation de leurs enfants.

Alors qu'une grande majorité de parents comptent maintenant sur le système scolaire pour contribuer à l'éducation de leurs enfants, la décision de faire l'éducation de ses enfants à domicile est devenu un choix très marginal, bien que présent internationalement. Par leur choix, ces familles expriment une conception de l'éducation qui ne se reconnaît pas dans notre système scolaire ou des attentes éducationnelles insatisfaites. Elles prennent aussi position de façon affirmée, quoique discrète, quant à la responsabilité, aux droits et au rôle privilégié des parents dans l'éducation de leurs enfants. Cet article présente les raisons du choix de l'éducation à domicile exprimées par les parents ayant participé à une enquête réalisée au printemps 2003, au Québec. L'enquête visait à documenter leurs conceptions de l'éducation et les raisons de leur choix, ainsi qu'à recueillir des informations sur l'expérience éducative et les caractéristiques sociodémographiques de ces familles.

\section{Problématique}

L'école à la maison[1], comme le disent les Québécois, serait l'équivalent de ce qu'on appelle en Europe l'éducation à domicile, ou du homeschooling américain, pratique éducative définie comme «l'éducation d'enfants d'âge scolaire sous la supervision de leurs parents, et elle remplace la fréquentation à temps plein d'un établissement d'enseignement.» ${ }^{[2]}$ (Lines, 1999, p. 1) Cette définition très large ne détermine que les personnes visées (enfants d'âge «scolaire»), puis procède par la négative (ils ne fréquentent pas l'école) pour ne préciser que le déplacement de la responsabilité de l'éducation des enfants, de l'État vers les parents. Ce sont, en effet, les seuls paramètres qui permettent de délimiter cette pratique, par ailleurs fort diverse.

Comme le souligne Pétrie (2001), les termes homeschooling et école à la maison suggèrent de fausses représentations, restrictives et souvent peu conformes à la réalité de cette pratique. Contrairement à ce que sous-entendent ces appellations, l'éducation à domicile n'est généralement pas confinée au domicile familial, non plus qu'elle ne tente toujours de reproduire le modèle scolaire. Les études visant à décrire cette 
pratique (Lines, 2000; Ray, 1994) dépeignent une forme d'éducation qui fait grand usage des richesses de la collectivité que sont les connaissances et les ressources des amis, du voisinage, de la parenté ainsi que celles des bibliothèques, des centres récréatifs et sportifs, des musées, des galeries d'arts, des commerces et organismes locaux, sans oublier, depuis quelques années, de l'Internet. Plusieurs familles font partie d'associations de parents-enseignants ou de groupes locaux visant la mise en commun des ressources éducatives et l'organisation d'activités de groupe pour les enfants. De plus, les méthodes d'enseignement ou d'accompagnement de l'apprentissage sont très variées, notamment au niveau de leur degré de structure extérieure à l'apprenant, s'échelonnant des cours par correspondance réalisés selon des horaires d'étude pré-établis au parcours d'éducation non formelle basé sur le dialogue, l'autodidaxie et l'expérience.

\section{L'éducation à domicile dans le monde}

Ce choix éducatif est celui de parents à travers le monde. Le Royaume-Uni, la NouvelleZélande et l'Australie ont de longues traditions d'éducation à domicile, certaines familles y ayant reçu leur instruction de cette façon depuis plusieurs générations (Meighan, 1995). Cette pratique est soutenue par des institutions comme le centenaire World-Wide Education Service (Boulter, 1989). L'éducation à domicile est présente également dans plusieurs autres pays d'Europe, comme la France, la Grèce, la Suisse, entre autres (Petrie, 2001). Ray (2001) rapporte aussi la présence de familles faisant l'éducation à domicile au Mexique, au Japon, en Russie et en Afrique du Sud.

Au Canada, en 2000-2001, de 60000 à 95000 enfants étaient éduqués à domicile (Ray, 2001). Selon les données disponibles, c'est aux États-Unis qu'on retrouve actuellement le plus grand nombre de familles faisant l'éducation à domicile: environ un million d'enfants seraient des homeschoolers (Lines, 2001), peut-être même 1,9 million (Ray, 2001). La croissance de la population de homeschoolers aux États-Unis a été documentée: les statistiques indiquent une augmentation annuelle de $10 \%$ à $24 \%$ depuis 1992 (Lines, 2001). Alors que les collèges et universités états-uniens accueillent depuis longtemps des étudiants ayant été instruits à domicile, au Canada, une récente invitation a été lancée aux homeschoolers par l'Université d'Ottawa [3].

\section{Au Québec : une pratique prévue dans la loi mais peu documentée}

$\mathrm{Au}$ niveau légal, l'éducation à domicile au Canada relève des différentes réglementations provinciales en matière d'éducation (Luffman, 1997). Au Québec, une dispense de l'obligation de fréquentation scolaire est prévue pour les enfants malades, handicapés, expulsés de l'école ou instruits à la maison. Cette dernière situation est décrite, depuis 1988, à l'article 15.4 de la Loi sur l'instruction publique: «Est dispensé de l'obligation de fréquenter une école l'enfant qui reçoit à la maison un enseignement et y vit une expérience éducative qui, d'après une évaluation faite par la commission scolaire ou à sa demande, sont équivalents à ce qui est dispensé ou vécu à l'école.» (L. R. Q., c. I-13.3).

Avant 2003, aucune enquête gouvernementale québécoise n'a été publiée sur la pratique de l'école à la maison au Québec et aucune étude scientifique n'a ciblé spécifiquement la population québécoise. Lorsqu'en 1997 Statistique Canada a tracé le profil de l'éducation à domicile au Canada, il était noté que, contrairement aux autorités scolaires des autres provinces et territoires du pays, «le ministère de l'Éducation du Québec (MEQ) ne recueille pas de données sur l'enseignement à domicile.» (Luffman, 1997, p. 45). Des entretiens avec des membres du comité légal de 
l'Association québécoise pour l'éducation à domicile (AQED) nous ont appris qu'une partie seulement des familles faisant l'école à la maison avisent leur commission scolaire ou le MEQ de leur intention de dispenser eux-mêmes l'instruction à leurs enfants. De plus, il n'y aurait pas consensus sur la façon de traiter ces dossiers et les informations ne seraient pas centralisées. Diverses commissions scolaires et divers directeurs d'école interpréteraient la loi provinciale de façons très différentes, donnant lieu à des attitudes très diversifiées dans leurs relations avec les parents-enseignants.

En novembre 2002, des membres de l'AQED exprimaient sur leur forum de discussion le souhait qu'une étude soit réalisée au Québec pour tracer un portrait de cette population et informer les décideurs comme les familles concernées. Or, le MEQ s'interroge actuellement sur l'ampleur du phénomène et compte prendre action pour baliser et préciser les dispositions de la loi. Par contre, même les provinces qui compilent ces informations mesurent difficilement le nombre d'enfants non inscrits à l'école, à cause de difficultés de recension. Par exemple, $8 \%$ des familles canadiennes que Ray (1994) a étudiées ont affirmé qu'elles n'étaient pas inscrites auprès des autorités éducatives. Aussi, $30 \%$ des familles ayant répondu à une enquête lors d'une conférence sur l'éducation à domicile de la Saskatchewan Home Based Educators (1997, cité par Luffman, 1997) ont dit n'avoir pas inscrit officiellement leurs enfants à l'enseignement à domicile.

L'absence de connaissances sur les familles faisant l'école à la maison au Québec n'est pourtant pas causée par l'absence de ces familles. Selon l'AQED, le Québec compterait actuellement entre 2500 et 5000 enfants éduqués à domicile, un nombre suffisant pour qu'on s'intéresse à leur parcours éducationnel. De plus, on peut supposer que leur nombre soit en croissance, puisqu'il l'est au Canada (Luffman, 1997), aux États-Unis (Lines, 1999) et dans plusieurs autres pays (Ray, 2001).

Le grand nombre de familles et leur présence sur tout le territoire de la province ne fait pas de doute après consultation des listes de groupes de soutien régionaux (des groupes de familles organisant entre elles des activités sociales et éducatives) et d'associations: l'Association québécoise pour l'éducation à domicile (AQED), l'Association chrétienne de parents-éducateurs du Québec (ACPEQ), la Home School Legal Defence Association (HSLDA) du Canada et la Canadian Association of Home Schoolers (CAHS). L'activité de ces familles est également tangible à travers les sites Internet familiaux, les entreprises commerciales qui leur offrent du matériel pédagogique, les conférences et symposiums organisés par leurs associations, les forums de discussions très fréquentés sur l'Internet, les commerçants et les institutions culturelles qui leur offrent des rabais, les entrevues et les émissions à ce sujet qui se multiplient dans les médias.

Néanmoins, il demeure que les parents qui optent pour ce mode d'éducation doivent accepter les aléas de la marginalité et une forme d'exclusion de leurs enfants de la majorité, évidemment scolarisée en institution. Ils peuvent rencontrer de l'incompréhension, voire des préjugés de la part de leur entourage et de certains administrateurs scolaires. En raison du flou réglementaire, la collaboration entre ces parents et les ressources du monde scolaire ne peut être présumée. Pour qu'ils acceptent cette situation, on peut être porté à croire que la décision des parents de faire l'école à la maison est un choix affirmé et qu'il repose sur des motivations assez fortes pour les mobiliser vers un mode de vie marginal, pour eux et pour leurs enfants. Mais quelles sont ces motivations qui poussent ces familles à se passer d'un service public qui leur est pourtant accessible gratuitement pour s'investir dans la prise en charge complète de l'éducation de leurs enfants? 


\section{L'école à la maison : les raisons derrière un choix marginal}

La conception des raisons du choix de l'éducation à domicile a évolué de portraits tels que ceux tracés par Mayberry (1989) et Van Galen (1988), où les catégories de raisons étaient étroitement liées à des catégories de parents, vers l'élaboration de hiérarchies ou de typologies de raisons du choix, comme dans une récente enquête nationale étatsunienne (Bielick, Chandler et Broughman, 2001) et dans le programme de recherche de Chapman et O'Donoghue (2000).

Van Galen (1988) a identifié deux types de parents qui éduquent leurs enfants à domicile, selon les raisons de leur choix. Les «idéologues» sont ceux qui rejettent l'école et le système public parce qu'ils sont en désaccord avec le contenu idéologique du programme d'études. Ils n'adhèrent pas aux valeurs transmises par l'institution scolaire et préfèrent avoir un plus grand contrôle sur l'éducation que reçoivent leurs enfants en leur offrant un mode de vie conforme aux valeurs de leur famille et à leur philosophie de vie. Les «pédagogues» regroupent les parents qui choisissent l'éducation à domicile parce qu'ils considèrent que certaines dimensions de l'environnement d'apprentissage scolaire sont néfastes ou ne conviennent pas à leur enfant. Cette catégorie comprend des parents d'enfants doués ou qui présentent des besoins éducatifs particuliers ainsi que des parents insatisfaits de la structure des institutions d'enseignement.

Mayberry (1989) nuance la typologie de Van Galen en proposant plutôt quatre groupes de parents. Parmi les parents qui éduquent leurs enfants à la maison pour des raisons idéologiques, elle distingue ceux qui sont motivés par des raisons religieuses de ceux qui aspirent à un mode de vie alternatif ou «Nouvel Âge». Elle divise aussi les «pédagogues» en deux groupes: ceux qui se préoccupent du cheminement scolaire de leurs enfants et ceux qui évoquent des raisons d'ordre sociorelationnel. Selon Mayberry, les parents qui éduquent leurs enfants à domicile pour des raisons religieuses proviennent de groupes chrétiens orthodoxes et voient dans ce mode d'éducation un moyen de transmettre à leurs enfants les principes judéo-chrétiens auxquels ils croient. Ils rejettent la laïcité de l'éducation publique et certains contenus du programme scolaire comme la théorie de l'évolution, l'éducation sexuelle et le relativisme moral. Les parents «Nouvel Âge» cherchent aussi à guider leurs enfants vers un système de valeurs auxquels ils adhèrent. Le besoin de développement spirituel de l'enfant et sa participation à l'ascension de l'humanité vers le «Nouvel Âge» dépendent de son éducation qui doit être conforme aux principes des philosophies métaphysiques invoquées.

Le troisième groupe identifié par Mayberry (1989) exprime des préoccupations pédagogiques liées au cheminement scolaire. Ils s'intéressent aux conditions favorisant la réussite scolaire de leurs enfants. Leurs critiques du système scolaire s'adressent au «nivellement par le bas» des exigences de l'enseignement à l'école et au peu de respect des différences individuelles dans les styles et les rythmes d'apprentissage. Ils croient que l'enseignement individuel et différencié qu'ils peuvent offrir à leurs enfants est gage d'une plus grande réussite scolaire et nourrira leur désir d'apprendre.

Préoccupés par d'autres dimensions pédagogiques de l'éducation, les parents attentifs au développement sociorelationnel de l'enfant sont insatisfaits de l'environnement d'apprentissage offert par l'école. Ils jugent que l'esprit de compétition, la pression exercée par les pairs, la séparation hâtive du milieu familial et certaines expériences sociales traumatisantes peuvent nuire au développement personnel et social des enfants. Ils croient que la famille offre un environnement qui favorise davantage l'apprentissage et l'épanouissement de l'enfant parce qu'il respecte mieux leurs besoins individuels, qu'il offre un style de vie plus détendu, plus sécurisant et plus 
aimant. Certaines de ces familles ne s'opposent aucunement à l'école et choisissent l'éducation à domicile pour le plaisir de vivre ensemble au quotidien, pour partager les moments de découverte avec leurs enfants et pour leur offrir un rythme d'apprentissage plus spontané et plus flexible.

Les catégories de parents basées sur les raisons de leur choix de l'éducation à domicile, telles que dégagées par Mayberry (1989) et Van Galen (1988), ont offert un premier éclairage et un cadre conceptuel aux études subséquentes sur les motivations des parents. Cependant, l'avancement des travaux sur ce sujet a déplacé l'intérêt porté à la définition de catégories de parents selon leurs motivations vers l'élaboration de hiérarchies ou de typologies de raisons du choix. Arai (2000), Welner (en arbitrage), Chapman et O'Donoghue (2000) et Marshall et Valle (1996) précisent que les typologies de Van Galen (1988) et de Mayberry (1989) ne représentent pas fidèlement toutes les familles. En effet, plusieurs familles glissent entre deux catégories parce qu'elles appuient leur choix de l'éducation à domicile sur une combinaison de raisons qui relèvent parfois de plus d'une catégorie. De plus, ces typologies ne révèlent pas l'évolution de l'opinion des parents qui, ayant d'abord choisi cette forme d'éducation pour des raisons d'une première catégorie, découvrent d'autres avantages de l'éducation à domicile et adoptent aussi des motivations d'une deuxième catégorie.

Dans un même ordre d'idées, Rothermel (1999) a décelé que la fréquentation d'autres familles et de regroupements d'éducation à domicile ainsi que les lectures faites sur le sujet influencent le discours des parents-enseignants, qui contient alors de plus en plus d'arguments relatifs aux philosophies de l'éducation. Dans un texte ultérieur (2002), elle note qu'une fois l'éducation à domicile amorcée, cette pratique devient de plus en plus un choix de mode de vie, alors que les motivations initiales cèdent la place à la découverte d'autres bénéfices de cette pratique. Qui plus est, les parents donnent parfois des raisons différentes pour chacun de leurs enfants: dans une étude nationale états-unienne (Bielick, Chandler et Broughman, 2001), parmi les 30 familles dans lesquelles le parent a répondu à l'enquête séparément pour chacun de ses deux enfants, 16 parents seulement ont donné exactement les mêmes raisons pour les deux enfants. Enfin, appuyant aussi la distinction à faire entre les catégories de parents et les catégories de motivations, Marshall et Valle (1996) mettent en lumière la tentation qu'amène la dichotomie de Van Galen de caricaturer ainsi les deux groupes: «Les Idéologues deviennent des fanatiques chrétiens d'extrême-droite et les Pédagogues deviennent des éco-progressistes Nouvel Âge» (p. 6).[4]

Une récente synthèse du phénomène de l'éducation à domicile en Amérique du Nord, réalisée par le Fraser Institute, avance que les familles pratiquant l'éducation à domicile au Canada ou aux États-Unis présentent généralement le même profil idéologique (Basham, 2001). Les Canadiens adopteraient ainsi la hiérarchie de motivations construite à partir d'une enquête nationale sur l'éducation réalisée pour le compte du gouvernement états-unien (Bielick, Chandler et Broughman, 2001) :

1. Pouvons offrir une meilleure éducation à la maison;

2. Raisons religieuses;

3. Environnement d'apprentissage pauvre à l'école;

4. Raisons familiales;

5. Pour développer le sens moral;

6. Objection à ce que l'école enseigne;

7. L'école n'offre pas de défi à l'enfant; 
8. Autres problèmes avec les écoles disponibles;

9. Problèmes de comportement de l'élève à l'école;

10. Enfant a des besoins spéciaux ou handicaps;

11. Transport/commodité;

12. Enfant pas assez vieux pour entrer à l'école;

13. Préférerions l'école privée mais n'en avons pas les moyens;

14. Carrière du parent;

15. N'a pas pu entrer à l'école de son choix;

16. Autres raisons (par exemple: choix de l'enfant, contrôle parental sur ce qui est appris, flexibilité, scolarisation à l'année) (p.11)[5].

Toutefois, une étude basée sur des entrevues auprès de 23 familles de l'Ontario et de la Colombie-Britannique sur les motivations des familles canadiennes à faire l'école à la maison (Arai, 2000) souligne plusieurs différences entre le discours des parents canadiens et celui des parents états-uniens. Contrairement à ce qu'il avait relevé dans les recherches antérieures, Arai a rencontré très peu de familles qui faisaient l'école à la maison par réaction à des expériences scolaires négatives ou dans le but de vivre un mode de vie alternatif. De plus, il n'a pas retrouvé la dichotomie présentée par le cadre conceptuel de Van Galen (1988). Les parents étudiés par Arai donnaient à la fois des raisons pédagogiques et des raisons idéologiques pour expliquer leur choix. Aussi, lors d'une étude auprès de 1000 familles canadiennes, réalisée en 1989 par Wendy Priesnitz, journaliste et fondatrice du CAHS, seul un quart des familles faisaient l'école à la maison pour des raisons religieuses, les autres ayant fait ce choix pour des raisons pédagogiques ou philosophiques[6] (Priesnitz, 1995).

Au même titre que les Canadiens semblent se démarquer des États-Uniens par les raisons du choix des familles pratiquant l'éducation à domicile, on peut supposer que la société québécoise, distincte par sa langue, sa culture et sa politique concernant l'éducation à domicile, puisse également se distinguer, non seulement des États-Uniens, mais aussi des autres Canadiens au chapitre des motivations des parents-enseignants. Peut-être leurs raisons se retrouveraient-elles dans un éventail plus large, comme celui colligé par Chapman et O'Donoghue (2000). Leur synthèse des résultats de la recherche sur les raisons du choix de l'éducation à domicile dégage neuf raisons principales évoquées dans les études recensées:

1. Insatisfaction envers les écoles traditionnelles;

2. Motifs religieux;

3. Affirmation que les écoles ne peuvent offrir aux enfants l'intérêt et l'attention individuels qu'ils peuvent recevoir dans leur famille;

4. Droits et responsabilités des parents prévalant sur les réglementations gouvernementales;

5. Protection des enfants contre des influences non désirées;

6. Expériences scolaires négatives;

7. Maintien de l'unité familiale;

8. Visions du développement de l'enfant et influences Nouvel-Âge (p. 24).[7] 
Chapman et O'Donoghue (2000) proposent un programme de recherche sur l'éducation à domicile qui contient des questions très pertinentes sur les raisons du choix éducationnel des parents. Ils suggèrent d'étudier le niveau d'importance de chacune des raisons généralement identifiées par la recherche dans la décision des parents, le niveau d'adhésion des parents à chacune, l'importance qu'ont pour les parents les différents facteurs de chaque catégorie de raisons, la relation entre chaque raison et la décision d'éduquer les enfants à domicile puis la satisfaction des parents envers l'alternative qu'ils ont choisie, au regard de chacune des raisons évoquées. C'est dans cet esprit qu'a été menée, de mai à juillet 2003, l'enquête québécoise présentée dans cet article.

\section{Méthodologie}

\section{Instrumentation}

À l'issue de la revue des écrits, aucun des questionnaires répertoriés visant à rendre compte des raisons motivant la pratique de l'école à la maison ne paraît couvrir, à lui seul, toute la gamme des possibles. De plus, la plupart des instruments limite les possibilités de réponse à un choix dichotomique (influence/n'influence pas) alors que, selon Chapman et 0'Dohoghue (2000), une mesure plus appropriée doit refléter le niveau d'importance de chacune des raisons et permettre la mise en relation des différentes raisons.

Pour mener à bien notre étude sur l'école à la maison au Québec, nous avons donc choisi d'élaborer notre propre instrument, qui comprend quatre sections: conceptions de l'éducation, raisons du choix, expérience de l'école à la maison et caractéristiques sociodémographiques. Ce questionnaire a été élaboré à partir d'un examen des questionnaires d'études spécifiques à l'école à la maison et d'enquêtes nationales étatsuniennes et néerlandaises sur l'éducation. De plus, afin de mieux cibler les préoccupations des familles engagées dans cette pratique et de générer des pistes d'exploration absentes des questionnaires, une analyse de contenu d'un forum de discussion portant sur l'école à la maison a aussi été réalisée en octobre 2002.

Les données traitées dans cet article proviennent de la section du questionnaire portant sur les raisons du choix de l'école à la maison. Cette section a été élaborée en compilant les motifs évoqués dans la littérature spécialisée et scientifique et dans les messages du Forum. Elle se compose de 50 énoncés proposant différentes raisons de choisir l'école à la maison. Les répondants sont invités à indiquer, sur une échelle de type Likert en cinq degrés (importance dans ma décision: aucune, faible, moyenne, grande ou très grande), à quel point chacune des raisons a contribué à leur décision d'entreprendre l'école à la maison et à maintenir leur décision. La liste de raisons suggérée et les réponses graduées permettent aussi d'obtenir une hiérarchie plus nuancée et d'éviter les réponses très larges comme celles qui dominent les hiérarchies du NCES (1999): «Pouvons donner une meilleure éducation à la maison» et de Chapman et O'Donoghue (2000): «Insatisfaction envers les écoles traditionnelles», raisons qui paraissent être l'envers et l'endroit d'une même affirmation fourre-tout.

Pour compléter l'éventail des raisons, une question ouverte (non analysée dans ce texte) est également placée à la fin de la liste, permettant au participant de noter d'autres raisons. La traduction du questionnaire en anglais a été vérifiée et améliorée par une enseignante d'anglais, bilingue, et ayant fait l'école à la maison. Une validation de contenu du questionnaire en français et en anglais a été effectuée à l'aide d'un comité-conseil bilingue constitué de parents-enseignants, de pionniers de regroupements de parents-enseignants et de communicateurs sur le sujet. 
Afin d'en favoriser une distribution aussi large que possible, le questionnaire a été produit en deux langues (français et anglais) et sur trois supports différents (papier avec distribution postale, html pour remplir en ligne et PDF pour impression à partir du site et acheminement postal), pour un total de six versions distinctes. Comme le site du questionnaire n'était pas référencié et qu'il était hébergé par le serveur de l'Université de Sherbrooke, seules les personnes ciblées par les chercheurs pouvaient vraisemblablement y avoir accès. Pour les réponses en ligne, l'identification de l'ordinateur d'origine (par les données sur le nom distant, l'utilisateur distant et l'agent http) a permis de s'assurer qu'aucun utilisateur ne puisse soumettre, sciemment ou par mégarde, des réponses multiples, sans toutefois l'identifier personnellement.

\section{Échantillonnage : une population sensible et inconnue}

La population des familles faisant l'école à la maison présente des difficultés d'échantillonnage puisqu'une partie importante de ces pratiques ne sont pas déclarées et qu'il n'existe aucune liste officielle des enfants non inscrits à l'école. Pour cette première exploration, nous avons constitué un échantillon aussi large que possible. Nous avons ainsi établi des ententes pour la distribution du questionnaire avec les responsables d'une association québécoise d'éducation à domicile, de groupes de soutien régionaux, de certains médias internes à cette population ainsi qu'avec un fournisseur de matériel didactique pour les parents-enseignants. Par leur intermédiaire, nous avons rejoint un certain nombre de familles qu'il est impossible de calculer à cause des multiples recoupements possibles entre les listes utilisées pour les rejoindre, de la désuétude de certaines coordonnées et de l'auto-exclusion des parents non-résidents du Québec ou ne pratiquant plus ou pas encore l'école à la maison.

Pour tenter de constituer un échantillon qui contiendrait, autant que possible, des individus issus de différentes tendances, nous nous sommes préoccupés de rejoindre des familles anglophones et francophones, des familles de toutes les régions du Québec, des familles de groupes religieux et de groupes non religieux. La collaboration de l'ACPEQ, qui compte 300 à 400 familles (La Presse, 17/12/2002) membres à vie depuis sa création, et du HSLDA du Canada nous a été refusée parce que leurs responsables s'impliquaient au même moment dans une étude pan-canadienne commandée par le HSLDA. Bien que nous ayons été informés que le MEQ avait obtenu récemment une liste d'enfants faisant l'école à la maison de la part des commissions scolaires, nous avons évité de solliciter sa collaboration pour ne pas éveiller la méfiance envers nos intentions de la part de certains parents non inscrits.

Le projet de recherche a été annoncé dans quelques médias électroniques spécialisés et des invitations à participer ont été diffusées par courrier électronique par des personnes-ressources du milieu. Pour respecter le désir de certains parents de ne pouvoir être retracés par les autorités scolaires, les envois postaux et électroniques ont été faits par les personnes-ressources, sans que nous ayons accès aux listes ni aux coordonnées des parents. Aucun rappel n'était donc possible. La version papier du questionnaire a été postée aux 520 membres présents et passés de l'AQED et elle a été incluse dans les 317 envois d'un magazine spécialisé entre le 9 et le 20 mai 2003. D'autres questionnaires ont été distribués à partir d'un kiosque tenu par notre équipe au Symposium de l'AQED le 24 mai 2003 et certains ont été postés à la suite de demandes adressées à l'équipe de recherche.

Cette distribution a permis de recueillir 203 questionnaires complétés (130 sur support papier et 73 réponses en ligne). De ce nombre, 47 ont utilisé un questionnaire en anglais (dont 9 en ligne) et 156 un questionnaire en français (dont 64 en ligne). Après vérification, il s'est avéré que 177 des 203 questionnaires provenaient de 
familles rencontrant entièrement le critère d'admissibilité à l'étude: être le parent d'au moins un enfant d'âge scolaire actuellement éduqué à la maison au Québec. Malgré ce critère, sept parents ont participé à l'étude en affirmant que leurs enfants, âgés de 0 à 5 ans, faisaient déjà l'école à la maison ${ }^{[8]}$. Trois parents prévoyaient commencer l'école à la maison à la rentrée 2003. Dix parents avaient terminé leur parcours d'éducation à domicile l'année scolaire précédant l'étude, soit en 2001-2002. Six autres parents avaient fait l'école à la maison dans les années antérieures à l'étude, mais les informations fournies ne nous permettent pas de savoir à quel moment, dans le parcours scolaire de leurs enfants (de trois, quatre, huit et neuf années de scolarité), ils avaient été éduqués à domicile. Afin de ne pas trop diminuer inutilement l'échantillon déjà restreint, et que rien ne porte à penser que les raisons pour faire l'école à la maison aient pu se modifier rapidement au cours des dernières années, tous les questionnaires reçus ont été retenus pour les analyses dont il est question dans le présent texte.

\section{Sexe, âge, expérience en éducation et niveau de scolarité des parents}

Tout comme dans l'Étude longitudinale du développement des enfants du Québec (Institut de la statistique du Québec, 2001), le questionnaire demande que ce soit «le parent qui connaît le mieux» la famille et les raisons du choix de l'école à la maison qui réponde. Nous appellerons ces parents «Parent 1». Parmi eux, 192 se sont identifiés comme des femmes, dix, comme des hommes et un n'a pas répondu à cette question. Le Parent 1 est âgé de 38 ans, en moyenne, avec un écart-type de 7 ans. Le «Parent 2», soit celui qui habite avec la famille et partage la responsabilité éducative, est âgé de 40 ans, en moyenne, avec un écart-type de 8 ans. Il est un homme dans 169 cas, une femme dans 11 cas et il n'y a pas de deuxième parent impliqué dans 23 familles. Le Parent $1 \mathrm{a}$ une expérience de formation ou de travail en éducation dans $44 \%$ des cas. Le plus haut diplôme d'études complété par l'un des parents est une maîtrise ou un doctorat chez 41 familles $(20 \%)$, un baccalauréat ou un certificat universitaire chez 78 familles (38\%), un diplôme d'études collégiales chez 34 familles (17\%) et un diplôme d'études secondaires complété ou non chez 50 familles (25\%). Ces données, dans la limite de la représentativité de l'échantillon, indiquent une sur-représentation des diplômés universitaires, des titulaires de maîtrises et de doctorats surtout, parmi les parents qui font l'école à la maison.

\section{Revenu familial et lieu de résidence familial}

Le revenu annuel familial médian des familles participantes se situe entre $40000 \$$ et $60000 \$ .10 \%$ des familles ont un revenu inférieur à $20000 \$, 32 \%$ le situent entre 20 $000 \$$ et $40000 \$, 30 \%$ entre $40000 \$$ et $60000 \$, 17 \%$ entre $60000 \$$ et $80000 \$, 8 \%$ entre 80000 et $100000 \$$ et $4 \%$ supérieur à $100000 \$$. Des familles de toutes les régions administratives du Québec, sauf de la Côte-Nord, ont participé à l'étude. Les régions qui comptent le plus de participants sont la Montérégie, la région de Montréal et l'Estrie, avec respectivement $20 \%, 19 \%$ et $13 \%$ des familles de l'échantillon. Les participants se répartissent presque également entre la ville (29\%), la banlieue (22\%), une petite ville ou un village $(22 \%)$ et la campagne $(27 \%)$. Considérant la taille de l'échantillon, on n'observe donc aucune sur-représentation ou sous-représentation notable quant à la répartition du revenu familial[9]. Il en est de même pour ce qui est du lieu de résidence, exception faite d'une certaine sous-représentation de la région de la Capitale-Nationale et d'une sur-représentation de l'Estrie dans l'échantillon[10]. 
Relation avec les autorités scolaires, langue d'enseignement, regroupements et engagement religieux ou spirituel

Les $40 \%$ des familles de l'échantillon ont affirmé que les autorités scolaires ne sont pas au courant de leur décision d'éduquer leurs enfants à domicile. Parmi les autres, 20\% ont une entente avec elles, $4 \%$ sont en conflit avec elles et $37 \%$ n'ont ni entente, ni conflit. La langue d'enseignement est le français chez $73 \%$ des familles, ce qui donne donc une large place aux familles anglophones, puisque $87 \%$ des enfants inscrits à l'école québécoise étudient en français (MEQ, 2003). Au plan des regroupements de familles faisant l'école à la maison, 129 familles sont membres de l'AQED, 93 participent à un groupe de soutien local, 46 sont membres de l'ACPEQ et 41 du HSLDA. Vingt-trois familles ne se disent membres d'aucun groupe et trois n'ont pas répondu à cette question. Plusieurs familles sont impliquées dans deux, trois ou quatre regroupements à la fois. Questionnées sur leur engagement religieux ou spirituel, 55 familles (28\%) affirment que la religion ou la spiritualité occupe une place fondamentale dans leur vie, $30(15 \%)$ disent qu'elle occupe une «certaine» place, 87 $(44 \%)$ valorisent une certaine forme de spiritualité mais n'ont pas d'engagement dans une religion organisée et 27 (14\%) n'ont aucun engagement religieux ou spirituel.

\section{Résultats}

\section{Distribution de l'importance accordée par les familles aux raisons}

Qu'elles soient classées selon la moyenne de l'importance accordée par l'ensemble des participants à chacune ou selon le nombre de participants leur ayant attribué une "grande» ou une «très grande» importance, les raisons s'organisent dans un ordre presque identique. Les tableaux 1 et 2 présentent les raisons auxquelles les familles, dans l'ensemble, accordent le plus et le moins d'importance.

Raisons les plus fréquentes

Tableau 1

\begin{tabular}{lccc}
\hline \multicolumn{1}{c}{ Raisons du choix ${ }^{11}$} & $\begin{array}{c}\text { Moyenne de l'importance } \\
\text { accordée } \\
\text { (échelle de 0 à 4) }\end{array}$ & Écart-type & $\begin{array}{c}\text { Pourcentage de familles } \\
\text { accordant une grande (3) ou } \\
\text { très grande (4) importance }\end{array}$ \\
\hline 7. enseignement individuel & 3,18 & 0,95 & $80 \%$ \\
4. projet familial & 3,18 & 1,07 & $77 \%$ \\
1. enrichissement & 3,12 & 0,99 & $76 \%$ \\
5. parents mieux placés & 3,03 & 1,04 & $72 \%$ \\
10. relations familiales & 3,02 & 1,03 & $73 \%$ \\
41. vie familiale et & 3,00 & 1,14 & $72 \%$ \\
communautaire & 2,98 & 1,23 & $72 \%$ \\
28. ratio adultes/enfants & & & \\
\hline
\end{tabular}

Raisons les moins fréquentes

\begin{tabular}{lccc}
\hline \multicolumn{1}{c}{ Raisons du choix } & $\begin{array}{c}\text { Moyenne de l'importance } \\
\text { accordée } \\
\text { (échelle de 0 à 4) }\end{array}$ & Écart-type & $\begin{array}{c}\text { Pourcentage de familles } \\
\text { accordant une grande (3) ou } \\
\text { très grande (4) importance }\end{array}$ \\
\hline 14. Dieu & 0,83 & 1,35 & $14 \%$ \\
50. accessibilité & 0,62 & 1,12 & $10 \%$ \\
48. activités enfant & 0,62 & 1,11 & $10 \%$ \\
37. pas prêt (cognitivement) & 0,53 & 1,08 & $9 \%$ \\
47. activités parent & 0,43 & 0,91 & $5 \%$ \\
49. maladie & 0,13 & 0,63 & $3 \%$ \\
\hline
\end{tabular}

$\mathrm{Au}$ chapitre des raisons moins fréquemment évoquées, mentionnons que certains enfants ne fréquentent pas l'école pour des situations de maladie, à cause d'activités extraordinaires de l'enfant (compétitions sportives, carrière artistique), parce que 
l'école de leur choix ne leur est pas accessible (temps de transport, coût de l'école privée, par exemple) ou parce que l'activité professionnelle des parents ne le permet pas. D'après nos résultats, ces situations existent mais demeurent exceptionnelles.

Pour six raisons, présentées au tableau 3, la distribution des participants révèle une polarisation: on observe que les plus grands nombres de réponses se retrouvent aux extrêmes de l'intervalle de choix de réponses, soit sous «aucune importance» et «très grande importance». De ces cinq raisons, trois sont reliées à la religion et trois sont liées à l'expérience et aux caractéristiques de l'enfant. Il faut donc comprendre que les faibles moyennes d'importance calculées pour ces items ne reflètent pas une tendance d'ensemble mais démontrent plutôt que des familles adhèrent entièrement à ces raisons alors que d'autres n'y adhèrent nullement, les premières étant moins nombreuses que les secondes dans les six cas.

Tableau 3

Raisons au sujet desquelles les familles sont polarisées

\begin{tabular}{lccccc}
\hline \multirow{2}{*}{ Raisons du choix } & \multicolumn{5}{c}{ Pourcentage des familles accordant une importance } \\
\cline { 2 - 6 } & aucune & faible & moyenne & grande & très grande \\
\hline Liées à la religion et à la spiritualité: & & & & & \\
11. religion & $\mathbf{5 0} \%$ & $13 \%$ & $12 \%$ & $7 \%$ & $\mathbf{1 8} \%$ \\
$\begin{array}{l}\text { 14. Dieu } \\
\text { 22. développement spirituel }\end{array}$ & $\mathbf{6 5} \%$ & $11 \%$ & $10 \%$ & $3 \%$ & $\mathbf{1 1} \%$ \\
\hline Liées à l'expérience et aux caractéristiques de l'enfant: & $\mathbf{2 4} \%$ & $18 \%$ & $15 \%$ & $15 \%$ & $\mathbf{2 7} \%$ \\
\hline 42. besoins spéciaux & $\mathbf{3 9} \%$ & $11 \%$ & $11,5 \%$ & $15,5 \%$ & $\mathbf{2 4} \%$ \\
34. expérience négative (enfant) & $\mathbf{4 4} \%$ & $10 \%$ & $12,8 \%$ & $10 \%$ & $\mathbf{2 5} \%$ \\
25. enfant transformé par l'école & $\mathbf{4 1} \%$ & $5 \%$ & $11 \%$ & $14 \%$ & $\mathbf{3 0} \%$
\end{tabular}

\section{Analyse factorielle}

Des analyses en composantes principales avec rotation varimax furent effectuées afin d'examiner la structure factorielle de la liste de raisons proposées. Une première analyse a extrait 14 composantes. Les quatre dernières raisons de la liste (47. activités parent; 48. activités enfant; 49. maladie; 50. accessibilité) furent retirées de ces analyses parce qu'elles étaient factuelles plutôt que subjectives et auraient pu être associées à n'importe laquelle des 46 autres raisons sans relation significative. L'analyse factorielle, qui extrayait alors 11 facteurs, a été forcée à 7 facteurs qui semblent mesurer chacun un construit théorique différent et expliquent $56,3 \%$ de la variance totale. La variance totale se répartit comme suit: $23,4 \%$ au premier facteur, $11,0 \%$ au deuxième, $6,4 \%$ au troisième, $5,5 \%$ au quatrième, $3,8 \%$ au cinquième, $3,2 \%$ au sixième et $3,0 \%$ au septième. Le tableau 4 rapporte la saturation des items sur chacun des facteurs. Ne furent retenus que les items ayant un coefficient de saturation supérieur à 0,30 et présentant une différence minimale de 0,10 avec leur coefficient de saturation sous un autre item (le cas échéant), selon les recommandations de Hair, Anderson, Tatham et Black (1998) et de Gorsuch (1983).

Les 7 facteurs dégagés de cette analyse sont: 1) Projet familial, 2) Objection au mode d'organisation sociale et pédagogique de l'école, 3) Enrichissement, 4) Souci du développement socioaffectif de l'enfant, 5) Transmission de valeurs religieuses, morales ou spirituelles, 6) Expérience scolaire négative de l'enfant et 7) Caractéristiques particulières de l'enfant. 
Tableau 4

Analyse factorielle en composantes principales avec rotation varimax

\begin{tabular}{|c|c|c|c|c|c|c|c|}
\hline \multirow[b]{2}{*}{ Items } & \multicolumn{7}{|c|}{ Facteurs } \\
\hline & 1 & 2 & 3 & 4 & 5 & 6 & 7 \\
\hline \multicolumn{8}{|l|}{ 1. Religion. morale et spiritualité } \\
\hline 11. religion & $0,840^{*}$ & & & & & & \\
\hline 14. Dieu & 0,793 & & & & & & \\
\hline 19. respect/discipline & 0,681 & 0,302 & & & & & \\
\hline 22. développement spirituel & 0,667 & & & & & & \\
\hline 13. contrôle sur l'éducation & 0,659 & & & & 0,317 & & \\
\hline 9. sens moral & 0,636 & & & & 0,315 & & \\
\hline 16. influences extérieures & 0,602 & & & & 0,575 & & \\
\hline 40. enseignants inconnus & 0,521 & & 0,379 & & & & \\
\hline 29. trop de règles/discipline & $-0,410$ & & & & 0,392 & & \\
\hline 12. philosophie & 0,399 & & & & 0,353 & & \\
\hline 35. expériences négatives (parent) & 0,381 & & & & & & \\
\hline \multicolumn{8}{|l|}{ 2. Projet familial } \\
\hline 4. projet familial & & 0,743 & & & & 0,309 & \\
\hline 3. liberté/flexibilité & & 0,741 & & & & & \\
\hline 41. vie familiale et communautaire & & 0,596 & & & & & \\
\hline 46. trop de temps à l'école & & 0,558 & & & & & \\
\hline 5. parents mieux placés & 0,378 & 0,555 & & & & & \\
\hline 15. poursuivre après 5 ans & & 0,535 & & & & & \\
\hline 18. responsabilité parentale & 0,482 & 0,509 & & & & & \\
\hline 10. relations familiales & 0,399 & 0,503 & & & & & \\
\hline 20. compétition/évaluation & & 0,462 & & 0,450 & & & \\
\hline 44. pas besoin de diplôme & & 0,392 & & & & $-0,322$ & \\
\hline \multicolumn{8}{|l|}{ 3. Organisation scolaire } \\
\hline 33. structure sociale & & & 0,717 & & & & \\
\hline 27. apprentissages non significatifs & & 0,335 & 0,699 & & & & \\
\hline 32. motivation à apprendre & & & 0,656 & & & & \\
\hline 23. nivellement par le bas & & & 0,561 & & & 0,466 & \\
\hline 39. méthodes d'enseignement & & & 0,543 & 0,382 & & & \\
\hline 26. conformisme & & 0,312 & 0,511 & 0,313 & & & \\
\hline 21. place aux parents & & 0,303 & 0,307 & & & & \\
\hline \multicolumn{8}{|l|}{ 4. Expérience scolaire négative } \\
\hline 34. expériences négatives (enfant) & & & & 0,834 & & & \\
\hline 25. transformé par l'école & & & & 0,752 & & 0,314 & \\
\hline 45. cadre scolaire & & & & 0,723 & & & 0,484 \\
\hline \multicolumn{8}{|l|}{ 5. Développement socioaffectif } \\
\hline 8. séparé longues périodes & 0,334 & & & & 0,533 & & \\
\hline 24. pression des pairs & 0,353 & & 0,308 & & 0,525 & & \\
\hline 28. ratio adultes/enfants & & & 0,356 & & 0,501 & & \\
\hline
\end{tabular}


(suite)

\begin{tabular}{|c|c|c|c|c|c|c|c|}
\hline \multirow[b]{2}{*}{ Items } & \multicolumn{7}{|c|}{ Facteurs } \\
\hline & 1 & 2 & 3 & 4 & 5 & 6 & 7 \\
\hline 30. dépendance affective & & & 0,369 & & 0,461 & & 0,304 \\
\hline 31. environnement violent & 0,306 & & 0,323 & & 0,459 & & \\
\hline 7. enseignement individuel & & & 0,308 & & 0,321 & & \\
\hline \multicolumn{8}{|l|}{ 6. Enrichissement } \\
\hline 6. avancé pour son âge & & & & & & 0,772 & \\
\hline 1. enrichissement & & & & & & 0,659 & \\
\hline 2. choix de l'enfant & & & & 0,334 & & 0,558 & \\
\hline 36. programme québécois & & & 0,386 & 0,405 & & 0,447 & \\
\hline 17. informations/stimulations & & 0,402 & & & & 0,404 & \\
\hline \multicolumn{8}{|l|}{ 7. Caractéristiques particulières } \\
\hline 38. pas prêt psychologiquement & & & & & & & 0,743 \\
\hline 37. pas prêt cognitivement & & & & & & & 0,694 \\
\hline 43. garçons & & & 0,356 & & & & 0,568 \\
\hline 42. besoins spéciaux & & & 0,324 & 0,399 & & & 0,453 \\
\hline Variance expliquée/facteur (total: $56,3 \%$ ) & $23,4 \%$ & $11,0 \%$ & $6,4 \%$ & $5,5 \%$ & $3,8 \%$ & $3,2 \%$ & $3,0 \%$ \\
\hline
\end{tabular}

Méthode d'extraction : Analyse en composantes principales.

Méthode de rotation : Varimax avec Normalisation de Kaiser.

La rotation a convergé en 17 itérations.

* Seuls les items présentant un coefficient supérieur à 0,300 et une différence minimale de 0,100 avec leur coefficient sous un autre facteur ont été conservés (en gras).

Comme le montre le tableau 5 qui suit, le degré d'intercorrélation entre les facteurs est généralement faible $(p<0,30)$ ou moyen $(0,30<p<0,60)$. Seuls les facteurs «Religion/morale/spiritualité» et «Développement socioaffectif» sont plus fortement corrélés $(p=0,603)$. On peut donc conclure à un bon niveau d'indépendance des facteurs entre eux.

Tableau 5

Intercorrélation des facteurs (khi-deux de Pearson)

\begin{tabular}{|c|c|c|c|c|c|c|c|c|}
\hline Facteurs & & 1 & 2 & 3 & 4 & 5 & 6 & 7 \\
\hline \multirow[t]{2}{*}{ 1. Projet familial } & $\begin{array}{l}\text { Corrélation de } \\
\text { Pearson }\end{array}$ & 1,000 & & & & & & \\
\hline & $\begin{array}{c}\text { Sig. (bicaudal) } \\
\mathrm{N}\end{array}$ & $\begin{array}{l}0,000 \\
202\end{array}$ & & & & & & \\
\hline \multirow[t]{2}{*}{ 2. Modes d'organisation } & $\begin{array}{l}\text { Corrélation de } \\
\text { Pearson }\end{array}$ & 0,482 & 1,000 & & & & & \\
\hline & $\begin{array}{l}\text { Sig. (bicaudal) } \\
\mathrm{N}\end{array}$ & $\begin{array}{c}0,000 \\
202\end{array}$ & $\begin{array}{c}0,000 \\
203\end{array}$ & & & & & \\
\hline \multirow[t]{2}{*}{$\begin{array}{l}\text { 3. Expérience scolaire } \\
\text { négative }\end{array}$} & $\begin{array}{l}\text { Corrélation de } \\
\text { Pearson }\end{array}$ & $-0,008$ & 0,277 & 1,000 & & & & \\
\hline & $\begin{array}{l}\text { Sig. (bicaudal) } \\
\text { N }\end{array}$ & $\begin{array}{c}0,906 \\
198\end{array}$ & $\begin{array}{c}0,000 \\
199\end{array}$ & $\begin{array}{c}0,000 \\
199\end{array}$ & & & & \\
\hline \multirow[t]{2}{*}{$\begin{array}{l}\text { 4. Développement } \\
\text { socioaffectif }\end{array}$} & $\begin{array}{l}\text { Corrélation de } \\
\text { Pearson }\end{array}$ & 0,583 & 0,534 & 0,130 & 1,000 & & & \\
\hline & $\begin{array}{l}\text { Sig. (bicaudal) } \\
\mathrm{N}\end{array}$ & $\begin{array}{c}0,000 \\
202\end{array}$ & $\begin{array}{l}0,000 \\
203\end{array}$ & $\begin{array}{c}0,067 \\
199\end{array}$ & $\begin{array}{l}0,000 \\
203\end{array}$ & & & \\
\hline \multirow[t]{2}{*}{ 5. Enrichissement } & $\begin{array}{l}\text { Corrélation de } \\
\text { Pearson }\end{array}$ & 0,314 & 0,338 & 0,296 & 0,182 & 1,000 & & \\
\hline & $\begin{array}{c}\text { Sig. (bicaudal) } \\
\mathrm{N}\end{array}$ & $\begin{array}{l}0,000 \\
202\end{array}$ & $\begin{array}{c}0,000 \\
202\end{array}$ & $\begin{array}{c}0,000 \\
198\end{array}$ & $\begin{array}{c}0,009 \\
202\end{array}$ & $\begin{array}{c}0,000 \\
202\end{array}$ & & \\
\hline
\end{tabular}




\begin{tabular}{|c|c|c|c|c|c|c|c|c|}
\hline Facteurs & & 1 & 2 & 3 & 4 & 5 & 6 & 7 \\
\hline & $\mathrm{N}$ & 202 & 203 & 199 & 203 & & & \\
\hline \multirow[t]{2}{*}{ 5. Enrichissement } & Corrélation de & 0,314 & 0,338 & 0,296 & 0,182 & 1,000 & & \\
\hline & $\begin{array}{l}\text { Sig. (bicaudal) } \\
\mathrm{N}\end{array}$ & $\begin{array}{c}0,000 \\
202\end{array}$ & $\begin{array}{c}0,000 \\
202\end{array}$ & $\begin{array}{c}0,000 \\
198\end{array}$ & $\begin{array}{c}0,009 \\
202\end{array}$ & $\begin{array}{c}0,000 \\
202\end{array}$ & & \\
\hline \multirow[t]{2}{*}{$\begin{array}{l}\text { 6. Religion/morale/ } \\
\text { spiritualité }\end{array}$} & $\begin{array}{l}\text { Corrélation de } \\
\text { Pearson }\end{array}$ & 0,377 & 0,210 & $-0,025$ & 0,465 & $-0,027$ & 1,000 & \\
\hline & $\begin{array}{l}\text { Sig. (bicaudal) } \\
\mathrm{N}\end{array}$ & $\begin{array}{c}0,000 \\
202\end{array}$ & $\begin{array}{c}0,003 \\
203\end{array}$ & $\begin{array}{c}0,729 \\
199\end{array}$ & $\begin{array}{c}0,000 \\
203\end{array}$ & $\begin{array}{c}0,707 \\
202\end{array}$ & $\begin{array}{c}0,000 \\
203\end{array}$ & \\
\hline \multirow[t]{2}{*}{$\begin{array}{l}\text { 7. Caractéristiques } \\
\text { particulières }\end{array}$} & $\begin{array}{l}\text { Corrélation de } \\
\text { Pearson }\end{array}$ & 0,193 & 0,203 & 0,260 & 0,261 & 0,014 & 0,098 & 1,000 \\
\hline & $\begin{array}{c}\text { Sig. (bicaudal) } \\
\mathrm{N}\end{array}$ & $\begin{array}{l}0,006 \\
202 \\
\end{array}$ & $\begin{array}{c}0,004 \\
202 \\
\end{array}$ & $\begin{array}{c}0,000 \\
198 \\
\end{array}$ & $\begin{array}{c}0,000 \\
202 \\
\end{array}$ & $\begin{array}{c}0,842 \\
202 \\
\end{array}$ & $\begin{array}{c}0,163 \\
202 \\
\end{array}$ & $\begin{array}{c}0,000 \\
202 \\
\end{array}$ \\
\hline
\end{tabular}

** La corrélation est significative au niveau 0,01 (bicaudal).

1. Projet familial : Ce facteur regroupe les énoncés qui communiquent un projet de vie familiale et communautaire, de découverte partagée, de relations familiales soutenues comme étant l'environnement le plus propice à l'éducation des enfants ainsi qu'au bonheur et à la liberté de tous les membres de la famille. Ce construit s'appuie aussi sur l'affirmation que les parents sont les mieux placés pour assurer l'éducation de leurs enfants, avant comme après l'âge de l'entrée à l'école. L'item qui dénonce la quantité de temps passé à l'école s'y retrouve aussi.

2. Objection au mode d'organisation sociale et pédagogique de l'école : Ce facteur est lié à des énoncés qui critiquent la structure scolaire. Y sont dénoncés le nivellement par le bas des programmes, les méthodes d'enseignement, l'encouragement du conformisme et le manque d'apprentissages significatifs. On y retrouve aussi les énoncés qui affirment que l'école détruit la motivation à apprendre et que son organisation sociale ne prépare pas les enfants à la vraie société.

3. Enrichissement : Ce facteur relie les énoncés qui portent sur la précocité de l'enfant, le désir d'offrir de l'enrichissement et de respecter le choix de l'éducation à domicile fait par l'enfant.

4. Souci du développement socioaffectif de l'enfant : Les raisons du choix reliées à ce facteur démontrent un souci de protéger et d'accompagner l'épanouissement socioaffectif de l'enfant. L'école à la maison est un moyen d'éviter l'environnement scolaire perçu comme trop violent, générateur d'une dépendance affective entre les enfants et d'une pression indue des pairs. La séparation trop longue d'avec les parents et le trop petit nombre d'adultes à l'école ajoutent à ce facteur de choix.

5. Transmission de valeurs religieuses, morales ou spirituelles : Les raisons qui correspondent à ce facteur expriment la volonté de donner à l'enfant une éducation et un mode de vie cohérents avec les valeurs religieuses, morales et spirituelles des parents. Les items mentionnant la religion et la parole de Dieu y sont reliés. Les préoccupations liées à ce facteur soulignent le besoin de développement spirituel de l'enfant, la difficulté de faire confiance à la compétence morale d'enseignants inconnus et l'absence de contrôle parental sur le contenu de l'éducation scolaire. La volonté de développer le sens moral de l'enfant et de transmettre des principes de respect et de discipline s'y retrouve, alors qu'une corrélation négative est observée avec l'item qui critique l'excès de règles et de discipline à l'école. Une faible corrélation $(\mathrm{k} 2=$ - 
0,025 et $p=0,729$ ) se présente avec les expériences scolaires négatives vécues par les parents.

6. Expérience scolaire négative de l'enfant : Les trois énoncés qui sont rattachés à ce facteur rapportent des expériences scolaires négatives, un malaise de l'enfant à l'intérieur du cadre scolaire et une transformation négative de l'enfant lors de la fréquentation scolaire.

7. Caractéristiques particulières de l'enfant : Ce facteur rassemble les raisons qui font état d'une inadéquation entre l'offre scolaire et l'enfant. On y lit un désir de retarder le début des apprentissages scolaires et l'éloignement du cadre familial ainsi qu'un besoin que l'école s'adapte aux garçons.

\section{Vue d'ensemble des raisons du choix}

Le rationnel qui sert de base à la décision d'une famille de faire l'école à la maison comporte plusieurs facteurs: en moyenne, 4 facteurs revêtent une importance de «moyenne» à «très grande» dans la décision d'une famille et 2 facteurs ont une importance de «grande» à «très grande». Il faudra donc éviter d'interpréter les catégories de raisons comme le reflet de catégories de familles.

Dans l'ensemble, les facteurs qui ont eu une importance de «moyenne» à «grande» dans la décision des parents sont, en ordre décroissant d'importance, le désir de vivre un projet familial, une objection au mode d'organisation sociale et pédagogique de l'école, une volonté d'enrichir la scolarité de leurs enfants et un souci de leur développement socioaffectif, tel que montré au tableau 6.

\section{Tableau 6}

Importance accordée à chaque facteur par l'ensemble des participants

\begin{tabular}{lccc}
\hline \multicolumn{1}{c}{ Facteurs des raisons du choix } & $\begin{array}{c}\text { Moyenne de } \\
\text { l'importance accordée } \\
\text { (échelle de 0 à 4) }\end{array}$ & Écart-type & $\begin{array}{c}\text { Pourcentage de } \\
\text { familles accordant } \\
\text { une grande (3) ou très } \\
\text { grande (4) importance }\end{array}$ \\
\hline Projet familial & 2,90 & 0,81 & $48 \%$ \\
Organisation scolaire & 2,63 & 0,89 & $35 \%$ \\
Enrichissement & 2,34 & 0,96 & $23 \%$ \\
Développement socioaffectif & 2,23 & 0,89 & $18 \%$ \\
Religion/morale/spiritualité & 1,94 & 0,89 & $14 \%$ \\
Expérience scolaire négative & 1,52 & 1,14 & $20 \%$ \\
Caractéristiques particulières de l'enfant & 0,94 & 1,23 & $4 \%$ \\
\hline
\end{tabular}

L'expérience scolaire négative est un facteur important pour presque $20 \%$ des familles. Toutefois, sa moyenne demeure faible, en partie à cause des réponses des familles dont les enfants n'ont jamais fréquenté l'école. Le facteur relatif à des préoccupations religieuses, morales ou spirituelles et celui qui exprime une inadéquation de l'offre scolaire avec les caractéristiques particulières de l'enfant demeurent minoritaires, malgré la polarisation des familles sur ces items. Une nuance intéressante a été apportée par des commentaires écrits d'un participant dans le questionnaire.

Alors que Rothermel $(1999,2002)$ a déjà montré que le rationnel à la base de la décision de débuter l'éducation à domicile peut se transformer avec l'expérience et le renouvellement du choix[11], une participante a mentionné que ses raisons différaient aussi selon l'âge de l'enfant concerné. Cette question pourra être approfondie dans des analyses subséquentes.Discussion 


\section{Discussion}

Il est d'abord observé que les raisons évoquées par les familles faisant l'école à la maison au Québec sont multiples et hétérogènes. Des 50 raisons proposées, chacune s'est avérée être d'une «très grande» importance pour au moins quatre familles et aucune n'a reçu l'assentiment général. L'éducation à domicile sert d'alternative à l'offre scolaire actuelle pour des familles d'horizons éducationnels très différents. On y retrouve une grande diversité de situations familiales, d'enfants, d'expériences, de projets et de convictions. En demandant aux familles de qualifier les énoncés de notre liste, nous avons pu dégager une logique interne à cette hétérogénéité sous la forme de sept facteurs motivant le choix de l'école à la maison. Ces facteurs nous apparaissent comme des construits théoriques distincts: 1) désir de vivre un projet éducatif familial, 2) objection au mode d'organisation sociale et pédagogique de l'école, 3) volonté d'enrichir la scolarité des enfants, 4) souci de leur développement socioaffectif, 5) expériences scolaires négatives, 6) transmission de valeurs religieuses, morales ou spirituelles, 7) observation d'une inadéquation entre l'offre scolaire et les caractéristiques particulières de son enfant.

Il est aussi observé que le rationnel décisionnel d'une famille est multidimensionnel. En moyenne, les familles de notre étude appuient leur décision sur deux à quatre facteurs qu'elles jugent importants parmi les 7 facteurs identifiés. Ces résultats corroborent ceux des recherches qui démontrent la complexité des rationnels décisionnels et le danger de chercher à associer des catégories de familles aux catégories de motivations (Arai, 2000; Welner, en arbitrage; Chapman et O'Donoghue, 2000 et Marshall et Valle, 1996). Cette structure factorielle établie empiriquement ouvre la voie à une typologie de raisons qui subdivise celles tracées par Van Galen (1988) et Mayberry (1989). Si l'on superpose les sept facteurs établis précédemment aux quatre catégories de Mayberry, des recoupements s'opèrent entre le facteur «religion/spiritualité/morale» et les raisons religieuses, entre le facteur «enrichissement» et les raisons pédagogiques. Cependant, les raisons pédagogiques peuvent être divisées entre le facteur «enrichissement», le facteur "caractéristiques particulières de l'enfant» et le facteur «objection aux modes d'organisation scolaire»[12]. Les raisons sociorelationnelles se trouvent aussi divisées en trois facteurs: «souci du développement socioaffectif de l'enfant», «projet familial» et «expérience scolaire négative».

Par ailleurs, les raisons idéologiques reliées à des philosophies «Nouvel Âge» (Mayberry, 1989) ne se dégagent pas de nos résultats. Sont-elles absentes au sein de l'échantillon? Moins présentes au Québec ? Ou l'instrument a-t-il failli à les distinguer ?[13] Si ces raisons idéologiques étaient présentes chez les familles de notre échantillon, peut-être se sont-elles fusionnées en certains points au facteur «projet familial» ou en d'autres points au facteur «souci du développement socioaffectif». Rappelons aussi les quatre raisons ayant trait à des situations familiales posant des problèmes d'ordre logistique: activités exceptionnelles de l'enfant ou des parents, difficultés d'accès à l'école de leur choix et maladie. Dans ces cas particuliers, il s'agit de raisons qui ne sont aucunement idéologiques ni pédagogiques.

L'importance relative accordée à chacune des raisons ${ }^{[14]}$ diffère sensiblement des résultats présentés par Chapman et O'Donoghue (2000) et par le gouvernement étatsunien (Bielick, Chandler, Broughman, 2001). Si l'on exclut de leurs classifications les raisons fourre-tout qui sont les plus fréquemment citées, comme: «Insatisfaction envers les écoles traditionnelles» et "Pouvons offrir une meilleure éducation à la maison», c'est le motif religieux qui arrive en tête de liste. Au Québec, les raisons directement liées à la religion ou à la parole de Dieu se situent au niveau le plus bas de 
l'ordre d'importance (voir tableau 2: raisons les moins fréquentes) et le facteur qui regroupe les préoccupations religieuses, morales et spirituelles ne revêt une «grande importance» que pour $14 \%$ de l'échantillon qui, dans l'ensemble, ne lui accorde qu'une importance de 1,85 (entre «faible» et «moyenne»). De plus, la proportion de familles faisant l'éducation à domicile pour des raisons religieuses est deux fois et demie plus petite que dans celle de Priesnitz (1989) pour l'ensemble du Canada. Or, ces statistiques ne peuvent être attribuées à une faible représentation des familles religieuses dans notre échantillon, puisqu'il comporte $23 \%$ de membres de l'ACPEQ (Association chrétienne de parents-éducateurs) et que 27\% des parents ont affirmé: «La religion ou la spiritualité occupe une place fondamentale dans ma vie». Il semble qu'au moment de faire des choix éducationnels pour les enfants, la religion ne figure pas nécessairement en premier, même lorsqu'elle joue un rôle d'avant-plan dans la vie spirituelle de la famille. Arai (2000), Marshall et Valle (1996) et Priesnitz (1995) ont tiré les mêmes conclusions de résultats semblables. Ici encore, la distinction entre les catégories de raisons et les catégories de parents s'impose.

Si les motivations religieuses sont moins présentes au Québec, ce sont les raisons reliées au projet familial qui prédominent. Dans l'étude du gouvernement états-unien (Bielick, Chandler et Broughman, 2001) et celle de Chapman et O'Donoghue (2000), ces raisons arrivent respectivement au quatrième et au septième rangs. Même si le thème de l'unité et des relations familiales est récurrent dans le discours sur l'éducation à domicile, la particularité de nos résultats tient à ce qu'aucune voix philosophique, politique ou religieuse ne semble dominer le discours québécois, ni même y avoir beaucoup de place. Il en va de même pour le rejet en bloc de l'intervention de l'État en éducation.

Au contraire du postulat de Basham (2001) à l'effet que les Canadiens adopteraient le même profil idéologique que les États-Uniens, les réponses de nos participants expriment des préoccupations différentes, en certains points majeurs, de celles des répondants à la plus récente enquête états-unienne sur l'éducation (Bielick, Chandler et Broughman, 2001). Comme dans l'étude d'Arai (2000), en Ontario et en ColombieBritannique, les familles québécoises de notre étude évoquent une combinaison de raisons idéologiques et pédagogiques. Les raisons qu'il décrit trouvent écho chez nos participants. Deux nuances se dessinent toutefois entre les résultats d'Arai et ceux de la présente étude, bien qu'il soit difficile de comparer les deux études en termes quantitatifs en raison des différences méthodologiques entre elles. L'importance des expériences scolaires négatives des parents dans leur choix de l'éducation à domicile semble proportionnellement plus grande dans notre échantillon et le désir de vivre un mode de vie alternatif[15] qu'il a entendu chez quelques participants n'a pas été exprimé dans nos résultats. Il faudra d'autres études locales pour ajouter à nos observations partielles des familles faisant l'école à la maison au Québec.

\section{Conclusion}

Les recherches sur l'éducation à domicile se heurtent toutes aux difficultés d'échantillonnage d'une population dont une partie préfère se tenir à l'écart de l'attention publique. Malgré les limites, en termes de représentativité de l'échantillon, les résultats présentés suggèrent des pistes d'interprétation permettant une première documentation partielle du phénomène de l'éducation à domicile au Québec et un début de compréhension du choix éducatif de ces familles. Dans cet article, nous avons brossé le portrait de familles pratiquant l'éducation à domicile, réparties sur le territoire québécois, dans les villes comme dans les campagnes, très scolarisées en moyenne et organisées en différents regroupements pour la mise en commun des 
ressources éducatives et le soutien entre parents. Dans l'ensemble, ces parents profondément engagés auprès de leurs enfants font principalement l'école à la maison pour poursuivre un projet d'éducation en famille et parce qu'ils portent un regard critique sur le système d'éducation, aux plans des modes d'organisation, du manque d'enrichissement des programmes et de l'impact de la scolarisation sur le développement socioaffectif des enfants.

Comme la recherche sur l'efficacité de l'éducation à domicile n'émet que des conclusions favorables sur la réussite scolaire et sociale des enfants concernés, cette pratique met les chercheurs en éducation et les autorités scolaires au défi de la comprendre et d'apprendre de cette vision marginale de l'éducation des enfants d'âge «scolaire». C'est dans l'optique d'une ouverture à ces questionnements, d'une meilleure compréhension de cette pratique et de ces familles qu'il faudra établir un dialogue avec ce mouvement éducatif et soutenir les parents dans leur travail d'éducation s'ils en expriment le besoin. La poursuite de l'analyse des réponses à notre enquête permettra de lire les besoins et les difficultés rencontrées par les familles, d'analyser leurs conceptions de l'éducation et de mieux les décrire. Nous tenterons ainsi de collaborer à la réflexion sur l'avenir et l'évolution des institutions éducatives à laquelle participent des chercheurs intéressés par l'éducation à domicile (Stevens, 2001; Meighan, 1995; Marshall et Valle, 1996; Rothermel, 2000): l'apport de leurs recherches sur la réalité vécue par les familles qui éduquent leurs enfants à domicile nourrit la discussion sur les fondements scientifiques qui soutiennent la scolarisation et ses modes d'organisation, sur la valorisation de la famille comme agent partenaire de la transmission des savoirs et de la culture d'une société et sur les partenariats possibles avec les parents-enseignants, comme l'offre de plus en plus populaire de fréquentation scolaire «à la carte».

\section{Notes}

[1]Les termes école-maison et école à la maison semblent être exclusivement québécois, alors que éducation à domicile, éducation au foyer, enseignement à la maison par les parents, enseignement à domicile, homeschooling, home schooling, home education, home-based education et home-based learning semblent utilisés pour nommer la même réalité ailleurs dans le monde. Ici, école à la maison sera utilisé dans un contexte québécois et éducation à domicile comme concept étudié.

[2]Traduction libre de: «the education of school-aged children under their parents' general monitoring, and it replaces full-time attendance at a campus school».

[3]The Ontario Federation of Teaching Parents. Canadian Admission Policies, [En ligne]. http://www.ontariohomeschool.org/canadmissionpolicies.html\#Ottawa (Page consultée le 18 septembre 2003).

[4]Traduction libre de: «Ideologues become right-wing Christian fanatics and Pedagogues become New Age eco-progressives».

[5]Traduction libre de: «1) Can give child beter education at home, 2) Religious reasons; 3) Poor learning environment at school; 4) Family reasons; 5) To develop character/morality; 6) Object to what school teaches; 7) School does not challenge child; 8) Other problems with available schools; 9) Student behavior problems at school; 10) Child has special needs/disability; 11) Transportation/convenience; 12) Child not old enough to enter school; 13) Want private school but cannot afford it; 14) Parent's career; 15) Could not get into desired school; 16) Other reasons*» «*[Par exemple:] It was the child's choice; to allow parents more control over what their children were learning; flexibility; and parents wanted year-round schooling.»

[6]Priesnitz y inclut des préoccupations que Mayberry (1989) appellerait «sociorelationnelles», comme l'inquiétude au sujet de la pression des pairs.

[7]Traduction libre de: «1) Dissatisfaction with traditional schools; 2) Religious motives; 3) The claim that schools cannot provide children with the personal interest and attention they can get from their family; 4) Parental rights and responsibility over government regulations; 5) Protection from 
unwanted influences; 6) Negative schooling experiences; 7) Maintenance of the family unit; 8) Views on child development; and New Age influences ».

[8]Il est intéressant de noter que la notion d'âge «scolaire» étant intimement reliée à la fréquentation scolaire, elle peut être ignorée ou redéfinie par les parents offrant l'éducation à domicile (en concevant l'éducation comme un processus continu à partir de la naissance, par exemple). Cette notion nous paraît aussi des plus flottantes parce qu'elle fait référence aux législations scolaires de chaque société et que les âges prescrits de début et de fin de la scolarité obligatoire ont été modifiés fréquemment dans l'histoire récente au Québec. Pour les familles poursuivant un projet d'éducation à domicile, il va de soi que ces âges charnières aient moins de signification.

[9]Si l'on compare ces données avec celles de l'ensemble des familles du Québec (Source: Statistique Canada, Tableau numéro 93F0029XDB96000, au catalogue de la série «Le pays»).

[10]Si l'on compare ces données avec celles de l'ensemble des familles du Québec (Source: Statistique Canada, Recensement du Canada, 2001, Compilation et traitement: Institut de la statistique du Québec, Direction de l'édition et des communications, 2002).

[11]C'est pourquoi le questionnaire demandait de qualifier l'importance que chaque raison avait eu à la fois dans leur décision initiale et dans la poursuite de l'école à la maison.

[12]Dans ce facteur, l'affirmation que la structure sociale de l'école ne prépare pas les enfants à la vie en société semble cependant appartenir davantage à la catégorie des raisons sociorelationnelles de Mayberry.

[13]Peut-être s'agit-il d'une indication que ces parents ont refusé de participer à l'enquête, mais cette explication est douteuse car il faudrait que ce sous-groupe, présent dans les autres études recensées, ait eu une attitude singulière pour la nôtre.

[14]On observe les mêmes distinctions, peu importe que les raisons soient regroupées par facteur ou non.

[15]Certains parents rencontrés par Arai (2000) associent le choix de l'école à la maison à un ensemble de valeurs qui leur dicte un mode de vie incluant le végétarisme, les médecines douces, des préoccupations environnementales et sociales. 


\section{Annexe}

\section{Énoncés de raisons du choix de l'école à la maison}

\section{Mots-clés}

1. enrichissement

2. choix de l'enfant

3. liberté/flexibilité

4. projet familial

5. parents mieux placés

6. avancé pour son âge

7. enseignement individuel

8. séparé longues périodes

9. sens moral

10. relations familiales

11. religion

12. philosophie

13. contrôle sur l'éducation

14. Dieu

15. poursuivre après 5 ans

16. influences extérieures

\section{Énoncés du questionnaire}

Nous pouvons offrir plus d'enrichissement à la maison.

C'est le choix de mon enfant et je le respecte.

J'ai fait ce choix pour la liberté et la flexibilité que cela donne à la famille.

L'école à la maison est un projet familial, pour le plaisir de vivre et de découvrir ensemble.

Les parents sont ceux qui connaissent le mieux leur enfant et qui sont les mieux placés pour s'engager dans son éducation.

Mon enfant est avancé pour son âge.

L'enseignement individuel ou en petit groupe est plus efficace et améliore l'apprentissage.

Je ne veux pas être séparé(e) de mon enfant pour d'aussi longues périodes de temps.

Je veux développer le sens moral de mon enfant.

J'accorde beaucoup d'importance aux relations parent-enfant et entre frères et soeurs.

Je désire transmettre à mon enfant des valeurs et un mode de vie conformes à ma religion.

Je désire transmettre à mon enfant des valeurs et un mode de vie conformes à ma philosophie13.

Je souhaite avoir davantage de contrôle sur ce que mon enfant apprend.

Dieu nous a donné des enfants, nous demande de les élever et de leur enseigner à respecter l'autorité des Saintes Écritures.

J'ai fait avec succès l'éducation de mon enfant entre 0 et 5 ans et je me sens capable de continuer.

Je désire protéger mon enfant des influences extérieures non désirées. 


\section{Mots-clés}

17. informations/stimulations

18. responsabilité parentale

19. respect/discipline

20. compétition/évaluation

21. place aux parents

22. développement spirituel

23. nivellement par le bas

24. pression des pairs

25. enfant transformé par l'école

26. conformisme

27. apprentissages non significatifs

28. ratio adultes/enfants

29. trop de règles/discipline

30. dépendance affective

31. environnement violent

32. motivation à apprendre

33. structure sociale

34. expérience négative (enfant)

35. expérience négative (parent)

36. programme québécois

\section{Énoncés du questionnaire}

En étant à l'extérieur de l'école, mon enfant a accès à plus d'informations et de stimulations.

L'éducation est ma responsabilité et non celle de l'État.

Je veux élever mon enfant dans le respect et la discipline.

La compétition et l'évaluation vécues à l'école nuisent à l'estime de soi et au respect du rythme de chacun.

L'école n'accorde pas assez de place aux parents.

L'école ignore le besoin de développement spirituel de l'enfant.

Le système scolaire effectue un nivellement par le bas en éducation, avec des programmes allégés.

À l'école, la pression des autres enfants nuit au développement de l'individu.

L'école avait transformé mon enfant. Il (Elle) ne s'épanouissait plus autant.

L'école encourage le conformisme.

À l'école, les apprentissages ne sont pas significatifs, ils sont décrochés de la réalité.

À l'école, le nombre d'enfants par adulte n'est pas approprié.

Il y a trop de règles et de discipline à l'école.

L'école crée une dépendance affective entre les enfants.

L'école est un environnement trop violent.

L'école détruit la motivation à apprendre.

La structure sociale de l'école ne prépare pas les enfants à la vraie société.

Mon enfant a vécu des expériences négatives à l'école.

J'ai personnellement vécu des expériences scolaires négatives et je ne veux pas y exposer mon enfant.

Le programme scolaire québécois ne correspond pas aux intérêts et aux forces de mon enfant. 


\section{Mots-clés}

37. pas prêt cognitivement

38. pas prêt psychologiquement

39. méthodes d'enseignement

40. enseignants inconnus

41. vie familiale et communautaire

42. besoins spéciaux

43. garçons

44. pas besoin de diplôme

45. cadre scolaire

46. trop de temps à l'école

47. activités parent

48. activités enfant

49. maladie

50. accessibilité

\section{Énoncés du questionnaire}

Mon enfant n'était pas cognitivement prêt pour les apprentissages scolaires à 5 ou 6 ans.

Mon enfant n'était pas psychologiquement prêt à quitter le cadre familial à 5 ou 6 ans.

Les méthodes d'enseignement de l'école ne me satisfont pas.

Je ne veux pas confier mon enfant à des enseignants dont je ne connais pas les valeurs et les compétences.

La vie familiale et communautaire est plus favorable que l'école au développement social et psychologique de l'enfant.

L'école ne répond pas suffisamment aux besoins spéciaux de mon enfant (besoin médical, difficultés de comportement ou d'apprentissage, douance).

L'école n'est pas faite pour les garçons.

On n'a pas besoin de diplôme pour réussir sa vie.

Mon enfant ne fonctionne pas bien dans un cadre scolaire.

Les écoliers passent trop de temps à l'école.

La fréquentation scolaire est incompatible avec mes activités professionnelles ou celles de l'autre parent.

La fréquentation scolaire est incompatible avec les activités particulières de mon enfant (compétitions, artiste).

Ce choix temporaire est dû à une maladie de l'enfant.

L'école de mon choix ne nous est pas accessible (réglementation, distance, coûts). 


\section{Bibliographie}

Arai, Bruce A. (2000), "Reasons for Home Schooling in Canada", Canadian Journal of Education, 25, 3, p. 204-217.

Basham, Patrick (2001), "Home Schooling: From the Extreme to the Mainstream", Public Policy Sources, 51, p. 1-18.

Bielick, Stacey, Chandler, Kathryn et Broughman, Stephen P. (2001), Homeschooling in the United States: 1999. (NCES 2001-033) U.S. Department of Education, Washington, DC, National Center for Education Statistics, 23 p.

Boulter, Hugh (1989), "The World-wide Education Service: 100 years of servicing parents as educators", Educational Review, 41, 2, p. 181-188.

Chapman, Anne et O'Donoghue, Thomas A. (2000), "Home schooling: an emerging research agenda", Education Research Perspectives, 27, 1, p. 19-36.

Gorsuch, Richard L. (1983), Factor Analysis (2e édition), Hillsdale, NJ, Lawrence Erlbaum Associates, $452 \mathrm{p}$.

Gouvernement du Québec (2003), Loi sur l'Instruction Publique, L. R. Q., c. I-13.3.

Hair, Joseph F., Anderson, Rolph E., Tatham, Ronald L. et Black, William C. (1998), Multivariate data analysis (5e édition), Upper Saddle River, N.J., Prentice Hall, 730 p.

Institut de la Statistique du Québec (2001), "Les nourrissons de 5 mois. Aspects conceptuels et opérationnels", Étude longitudinale du développement des enfants du Québec (ÉLDEQ 1998-2002), Sainte-Foy, ISQ.

Lines, Patricia M. (2001), "Homeschooling", ERIC Digest, 151.

Lines, Patricia M. (2000), "Homeschooling comes of age", Public Interest, Été, Washington.

Lines, Patricia M. (1999), "Homeschoolers: Estimating Numbers and Growth", National Institute on Student Achievement, Curriculum, and Assessment, Office of Educational Research and Improvement, U. S. Department of Education. [En ligne], http://mirror.eschina.bnu.edu.cn/Mirror/ed.gov/www.ed.gov/offices/OERI/SAI/homesch ool/ (Page consultée le 16 septembre 2003).

Luffman, Jacqueline (1997), "Profil de l'enseignement à domicile au Canada", Revue trimestrielle de l'éducation, 4, 4, p. 30-47.

Marshall, Dan J. et Valle, James P. (1996), "Public School Reform: Potential Lessons from the Truly Departed", Education Policy Analysis Archives, [En ligne] 4, 12. http://epaa.asu.edu/epaa/v4n12.html (Page consultée le 16 septembre 2003).

Mayberry, Maralee (1989), "Home-based Education in the United States: demographics, motivations and educational implications", Educational Review, 41, 2, p. 171-179.

Meighan, Roland. (1995), "Home-based Education Effectiveness Research and Some of its Implications", Educational Review, 47, 3, p. 275-287.

Ministère de l'Éducation du Québec (2003), Regards statistiques sur l'éducation, Sommaire 1997-1998 à 2001-2002, [En ligne], www.meq.gouv.qc.ca/stat/Stat_fr/s_ele6.htm, (Page consultée le 1 décembre 2003).

Petrie, Amanda (2001), "Home Education in Europe and the Implementation of Changes in the Law", International Review of Education, 47, 5, p. 477-500.

Priesnitz, Wendy (1995), School Free/ The Home Schooling Handbook, St. George, ON, The Alternate Press, 143 p.

Ray, Brian D. (2001), "Homeschooling in Canada", Education Canada, 41, 1, p. 28-31. 
Ray, Brian D. (1994), A Nationwide Study of Home Education in Canada: Family Caracteristics, Student Achievement, and Other Topics, Salem, OR, National Home Education Research Institute, 101 p.

Rothermel, Paula (2002), "Home-Education: Aims, Practices and Outcomes", Paper presented at the Annual Conference of the British Educational Research Association, University of Exeter, England, 12-14 September 2002. [En ligne], http://www.jspr.btinternet.co.uk/Research/Researchpaper/abstracts.htm (Page consultée le 16 septembre 2003).

Rothermel, Paula (2000), "The Third Way in Education: Thinking the Unthinkable", Education 3-13, 28, 1, March. Staffordshire, Threntham Books.

Rothermel, Paula (1999), "A nationwide study of home education: early indications and wider implications", Education Now, Été. [En ligne], 24. http://www.jspr.btinternet.co.uk/Research/Newspaper/ednow.htm. (Page consultée le 16 septembre 2003).

Van Galen, Jane (1988), "Ideology, curriculum and pedagogy in home education", Education and Urban society, 21, 1, p. 52-68.

Welner, Kariane Mary (en arbitrage) "Understudied Education: Toward Building a Homeschooling Agenda." [En ligne], http://edtech.connect.msu.edu/Searchaera2002/viewproposaltext.asp?propID=5052

(Page consultée le 16 septembre 2003). 